\title{
EXTRACTING FEATURES OF TRANSIENT ELECTRIC FIELDS WITH FOURIER AND WAVELET TRANSFORM -A CASE STUDY OF LIGHTNING POSITIVE RETURN STROKE Shriram Sharma*
}

*Department of Physics, Amrit Campus, Tribhuvan University, Nepal, and South Asian Lightning Network (SALNet).

\begin{abstract}
Frequency domain information were extracted from the time domain electric fields pertinent to the lightning positive return strokes applying Fourier transform and Wavelet transform. The electric field radiated by positive ground flashes striking the sea were recorded at $10 \mathrm{~ns}$ resolution at a coastal station to minimize the propagation effects. The frequency spectrum of the electric field of positive return strokes were computed applying the Fourier transform technique in the range of $10 \mathrm{kHz}$ to $20 \mathrm{MHz}$ owing to the fact that this range of frequency is of very much interest to the researchers and design engineers. The amplitude of the energy spectral density decreases nearly as $f^{-1}$ from $10 \mathrm{kHz}$ to about $0.1 \mathrm{MHz}$ and drops nearly as $f^{-2}$ up to $8 \mathrm{MHz}$. Applying the wavelet transform technique, the same positive return strokes are found to radiate in the frequency range of 5.5 to $81 \mathrm{kHz}$ with the average spread distribution of 13.6 $\mathrm{kHz}$ to about $30 \mathrm{kHz}$. From frequency spectrum obtained from the Fourier transform it is difficult to identify as which phase of the return stroke radiates in the higher frequency range and that in the lower frequency range, whereas, one can easily identify from the frequency spectrum obtained with the wavelet transform that ramp portion of the positive return stroke radiates in the larger spectral range as compared to that of initial peak of the return stroke. Also, from the spectral density map obtained from wavelet transform one can easily observe the contribution of each phase in a range of frequency, which is not possible from the Fourier transform technique. Clearly, the wavelet transform is much more powerful tool to extract the frequency domain information of a non-stationary signal as compared to that of Fourier transform.
\end{abstract}

Key words: Lightning; Fourier; Wavelet; Frequency-spectrum.

\section{INTRODUCTION}

Lightning is a very common atmospheric electrical discharge that takes place between two electrically imbalanced regions. The primary source of the lightning discharge are the cumulonimbus clouds called thunderclouds, though lightning activities have been observed in the volcanic eruption, tornados, wind storms etc. Irrespective of the source, lightning is one of the major natural hazards that kills over 24,000 people each year globally, leaving 3 folds of people injured ${ }^{1}$. Lightning causes tremendous loss to the physical property transmission on and communication system are being the worst victims.

Such losses can be caused not only by direct lightning strikes to the objects but also by the indirect strikes. During the direct strike, the huge current flowing in the lightning channel is the main cause of damage whereas, the electric and magnetic fields produced by the lightning channel is the main indirect source of damage. The electric and magnetic fields radiated by lightning normally

Author for Correspondence: Shriram Sharma, Department of Physics, Amrit Campus, Tribhuvan University, and Nepal, South Asian Lightning Network. Email: ramhome2@hotmail.com 
interact/couple with the physical structures resulting in the change in potentials that in turn, excites the structures. The extent of excitation depends upon the closeness of the wavelength of electromagnetic field to the dimension of the object. It is therefore, the frequency domain information pertinent to the lightning electromagnetic fields is of great importance in order to design appropriate protective system against lightning hazards. The frequency spectrum of the electromagnetic field generated by the lightning is of much interest both for engineering assessments and scientific investigations. The frequency range of $1 \mathrm{MHz}$ to $20 \mathrm{MHz}$ is of more concern, for this range corresponds to the natural frequencies of the most of the physical structures, particularly those structures which have the dimensions of about 10 to $100 \mathrm{~m}^{2,3}$. The use of the solid state devices in the airborne vehicles has increased the threat due to electromagnetic radiation from lightning. These devices when coupled with the radiation of the resonant frequency can upset the system or permanently damage the electronic system. In fact, lightning discharges are the principal cause of deleterious excitations to the electronic systems and hence from practical point of view it is important to understand in detail, amplitude spectrum of the lightning ${ }^{2}$.

The measurement of the frequency spectrum in a lightning flash, which are of much interest in understanding the physics of lightning, have been made either by monitoring the power received at individual frequencies using a narrow bandwidth recording device or by recording the transient radiation with wide bandwidth device and then Fourier transforming the waveform to obtain a spectrum ${ }^{4}$. The measurements of first type were extensively used in 1950's and 1960's however that of second type were mainly used after $1980{ }^{4}$. The frequency spectra obtained from both the ways were found to be in good agreement at low frequencies to about $1 \mathrm{MHz}$. However, the spectra obtained from narrowband measurement exhibit a great spread above $1 \mathrm{MHz}^{5}$. The Fourier transform approach, widely used after $^{6}$, has an advantage that a spectrum can be associated with a particular lightning process with the shape of the waveform ${ }^{3}$. Although the Fourier transform technique has advantages over the narrow band techniques it has limitations too. When a time domain signal is Fourier transformed to frequency domain signal the time information is lost. This issue is not important while dealing with the stationary signals but while dealing with the transient signals, as that of lightning electromagnetic fields, it becomes significant. The Fourier transform approach, widely used after Sehran et al. $^{6}$, has an advantage that a spectrum can be associated with a particular lightning process with the shape of the waveform ${ }^{3}$.

Sehran et al. ${ }^{6}$, analyzing the Fourier transformation of the first and subsequent return strokes reported that the frequency spectrum falls off nearly as $f^{-1}$ between the 5 $\mathrm{kHz}$ to $100 \mathrm{kHz}$. They have further reported that the trend for the subsequent return strokes is also same but with somewhat low amplitude. Weidman et al. ${ }^{2}$, analyzing the spectra of first return strokes, measurement being carried out almost over the salt water to minimize the propagation effect, reported that $f^{-1}$ trend can be extended up to 2 MHz. However, the trend changes as $f^{-2}$ between 2 $\mathrm{MHz}$ to $10 \mathrm{MHz}$ and as $f^{-5}$ above $10 \mathrm{MHz}$. Weidman et al. ${ }^{2}$ further reported that spectra of the fast rising portion of leader steps, the initial fast transition in return strokes and transition in positively intra-cloud pulses to be surprisingly similar.

Weidman and Krider ${ }^{7}$, analyzing the amplitude spectra of the fast rising, initial portion of fields produced by return strokes, leader steps and cloud pulses in the range of 1 $\mathrm{MHz}$ to $20 \mathrm{MHz}$, reported that the spectrum amplitude varies as $f^{-1}$ from $1 \mathrm{MHz}$ to $6 \mathrm{MHz}$ and it varies as $f^{-2}$ from $6 \mathrm{MHz}$ to $20 \mathrm{MHz}$. They further reported that the spectral amplitudes of leader step just before return strokes and the fast portion of cloud pulses that triggered the recording system tend to lay 5 to $10 \mathrm{~dB}$ below the amplitudes of first return strokes over the entire frequency interval.

To understand the temporal features of the frequency content of the different events of lightning wavelet 
transform has been used in this study. The wavelet transforms have been used in various geophysical studies in the $1990 \mathrm{~s}^{8}$, however they have not been widely used in the analysis of lightning electromagnetic fields except for $9,10,11,12,13,14$ for different purposes in the field of lightning activities. Using wavelet transform one is able to determine the dominant modes of variability and how those modes vary in time ${ }^{8}$. More recently in an extensive work, Sharma et $\mathrm{al}^{15}$ obtained the frequency spectra of various lightning phenomena making comparison between frequency contents of each phenomenon using the wavelet transform. Sharma et. al. ${ }^{15}$ found that the lightning ground flashes are the main source of very low and low frequency range of about $2.8 \mathrm{kHz}$ to about $81 \mathrm{kHz}$, whereas the initial breakdown activities, leader stages and narrow bipolar pulses radiate in the medium frequency range of about $50 \mathrm{kHz}$ to about $740 \mathrm{kHz}$.

In this study, the time domain electric field radiated by the positive return strokes have been analyzed by using both Fourier transform and wavelet transform technique with an objective of extracting the frequency content in the signal and to compare the efficiency of the two techniques. Furthermore, lightning positive ground flashes are not very well understood as compared to those of negative ground flashes and hence the positive ground flashes have been selected in this study.

\section{MEASUREMENT AND DATA ACQUISITION}

The electric field signatures analyzed in this study were measured in Fanö Island, at the west coast of Denmark. The measuring station was located a few tens of meters from the Atlantic Ocean. Locations of flashes were obtained using the data provided by the Danish Lightning Location System. The vertical electric field was sensed with a parallel plate antenna and the signals from the antenna system were recorded by LeCroy 9350L, oscilloscope working in pre-trigger mode with a sampling frequency of $100 \mathrm{MS} / \mathrm{s}$. The electric field sensed by the antenna was transferred to the oscilloscope through an electronic circuit including a buffer amplifier (LH0033) with a $60 \mathrm{~cm}$ long RG58 cable. The details of the circuit have been given by Sharma et al. ${ }^{16}$. The electric field due to positive return strokes, presented in this work were recorded at a sampling interval of $10 \mathrm{~ns}$ and the sampling frequency of $100 \mathrm{MS} / \mathrm{s}$ for a period of $50 \mathrm{~ms}$. The Nyquist frequency of the data acquisition is $50 \mathrm{MHz}$.

\section{ANALYSIS AND RESULTS}

The electric field due to positive return strokes were range normalized to $50 \mathrm{~km}$ assuming the inverse distance dependence of the radiation field. In order to obtain the frequency spectrum of only the return stroke activity, a part of the record consisting of the return stroke has been selected from the whole record.

For this reason and for the sake of uniformity we have selected the data with time window of $81.92 \mu \mathrm{s}$. This window length is sufficient to cover much of the return stroke activity of every record under study but for the long impulse currents which has a longer duration of a millisecond range ${ }^{17,18}$.

The selected window covering the return stroke activity of one of the return strokes has been depicted in figure 1 .

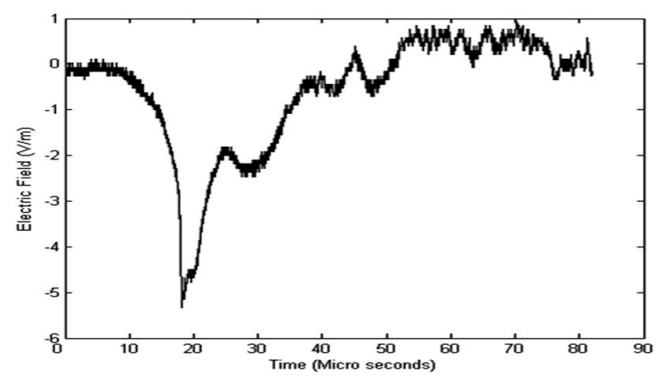

Figure 1: The expansion of the selected window of one of a positive return stroke under study. Some of the leader type pulses can be seen in the tail part of the stroke.

It is customary that the selected part of the stroke would not end in phase and hence the spectra would result in a leakage. In order to reduce any leakage of the energy in computing the Fourier transform, the selected wave structure has been applied with the well-known Hanning window.

The windowed fields were then computed by a discrete Fourier transform (DFT) algorithm. The DFT of a function $E(t)$ is given by;

$\mathrm{F}(\mathrm{k} \Delta \mathrm{f})=\Delta \mathrm{t} \sum_{\mathrm{n}=0}^{\mathrm{N}-1} \mathrm{E}(\mathrm{n} \Delta \mathrm{t}) e^{-(i 2 \pi k \Delta f)(n \Delta t)}$ 
Where, $\mathrm{k}=0,1,2 \ldots \mathrm{N}-1 .(\Delta \mathrm{t}=$ time increment or time resolution, $\Delta \mathrm{f}=$ frequency increment or frequency resolution. $\Delta \mathrm{f}=1 / \mathrm{T}$; $\mathrm{T}$ is the total sampling time).

Energy spectral density (ESD) has been calculated using the expression given by equation (2).

$$
E S D(f)=0.5[2 \pi \Delta t F(f)]^{2}(\mathrm{~V} / \mathrm{m} / \mathrm{Hz})^{2} .
$$

To correct the loss of energy as an average effect of the Hanning window the energy spectral density is multiplied by a factor of $8 / 3$. The amplitude of the ESD is taken on base 10 logarithmic scale to obtain meaningful spectra in decibels $(\mathrm{dB})$.

$E_{s}=10 \log _{10}\left(10^{E_{t} / 10}-10^{E_{n} / 10}\right)$

Where $E S$ is the energy contribution due only to the signal, $E_{t}$ is energy contribution due to the both signal and the noise and $E n$ is the energy contribution by the noise only.

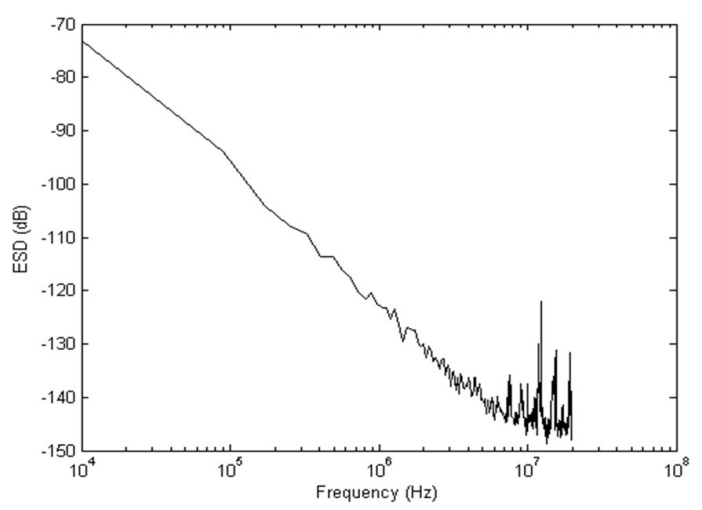

Figure 2: An average energy spectrum of 23 positive return strokes. The amplitude varies nearly as $1 / \mathrm{f}$ up to about 0.1 MHz and nearly as $1 / \mathrm{f}^{2}$ up to about $8 \mathrm{MHz}$ and constant afterwards with a few peaks at 12, 15 and $19 \mathrm{MHz}$.

An average energy spectrum of 23 positive return strokes after subtracting the noise contribution from each has been computed using ensemble averaging and is shown in figure 2 .

The amplitude of the average energy spectral density of the positive return strokes apparently decreases as $f^{-1}$ from $10 \mathrm{kHz}$ to $0.1 \mathrm{MHz}$, nearly as $f^{-2}$ from $0.1 \mathrm{MHz}$ to about $8 \mathrm{MHz}$ and apparently remains constant afterwards with a few peaks at $12 \mathrm{MHz}, 15 \mathrm{MHz}$, and $19 \mathrm{MHz}$.

Since the wavelet transform has been applied in various studies and its mathematical description can be found in those studies and the references therein, they are not further discussed here. But for the sake of general idea, a brief discussion is presented here.

The wavelet transform decomposes a time domain signal into time-frequency space. As the Fourier analysis is a breaking up of a signal into the infinite functions of sine and cosine waves, the wavelet analysis is the breaking up of a signal into shifted and scaled versions of ' $a$ ' original (or mother) wavelet ${ }^{14}$. The wavelet analysis can also be viewed as an evaluation of the similarity indices between the signal and daughter wavelets $(\psi)$ located at time position $b$ with scale ' $a$ ' as it is equivalent to a scalar product of the signal itself and the daughter wavelets (A. Graps 1995). A signal with a set of finite functions is called a wavelet $\psi_{0}(t)$ which is expanded $\psi_{0}(a t)$ and translated $\psi_{0}(t+b)$ into new versions called daughter wavelets.

The continuous wavelet transform of a discrete sequence $x(t)$ is defined as the convolution of $x(t)$ with scaled and translated version of $\psi_{0}(t)$ given as

$W_{t}(a, b)=\sum_{t=0}^{N-1} x(t) \psi *\left[\frac{(t-b) \delta t}{a}\right]$

Where ' $\boldsymbol{b}$ ' is the translation parameter in time space and ' $\boldsymbol{a}$ ' is the dilation parameter also known as Scale. Also, the (*) indicates the complex conjugate.

In this study, out of various wavelets, Derivative of Gaussian (DOG) wavelet was chosen though the type of wavelet function is not critical for the computation of wavelet power spectrum ${ }^{9}$. Nevertheless, the DOG is the real valued wavelet and captures both the positive and negative oscillations of the time series as separate peaks in wavelet power ${ }^{8}$. Also, the DOG wavelet is narrower in time space but broader in spectral space. Moreover, different wavelets were applied to the same lightning signal and it was observed that the results diverge a lot when dealing with orthogonal wavelets, the results diverge 
from type to type of mother wavelet Results were also found to be diverged for the same mother wavelet but with different parameters. Computation was done directly with the Matlab tool kit package. However, an analysis of the results obtained from the computation using nonorthogonal wavelet shows that the divergence of the results is much less. This stability becomes much significant when the DOG wavelet (Mexican Hat with $\mathrm{m}=$ 2 ) is applied. The continuous wavelet transform computation of a discrete signal was done with the routine given by Torrence and Compo $(1998)^{8}$.

The mathematical expression for this kind of wavelet is given by equation (5).

$$
\psi_{0}(t)=\frac{(-1)^{m+1}}{\sqrt{\Gamma\left(m+\frac{1}{2}\right)}} \frac{d^{m}}{d t^{m}}\left(e^{-t^{2 / 2}}\right)
$$

Where $\mathrm{m}=2$ for Derivative of Gaussian, also called Mexican hat wavelet. The wavelet power spectrum can be defined as the square of the magnitude of the wavelet transform given by Torrence and Compo $; \quad\left|W_{t}(a, b)\right|^{2}$. We have also performed the significance test in order to check the significance of wavelet power spectrum over the background noise spectrum (generally white noise and red noise) to a confidence level of $95 \%$. (It is to be noted that the white noise is a signal, analogous to the white light, which produces flat frequency spectrum, whereas the red noise, analogous to the random walk, produces frequency spectrum that varies as $1 / \mathrm{f}^{2} .{ }^{15}$

The cone of influence has also been computed in order to address the edge effects as was done by Sharma et. $\mathrm{al}^{15}$.
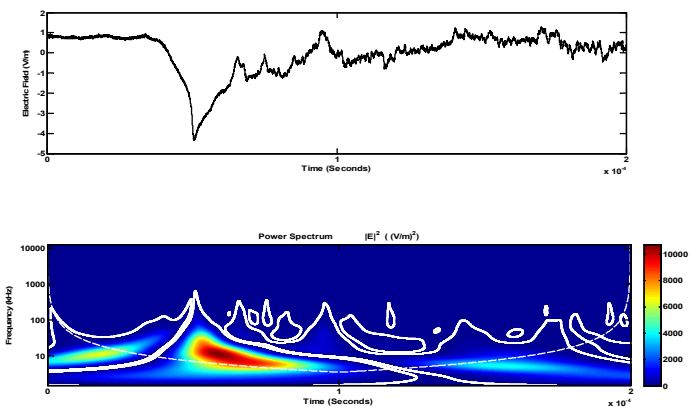

Figure 3: Wavelet power spectrum of a positive return stroke same as in figure 1. The energy radiated by the small pulses superimposed in the later part of the ramp is insignificantly low and hence their contribution is overwhelmed by the low frequency component of the radiation.

Depicted in Table 1 is the statistics of the wavelet power spectra of 23 positive return strokes in which the average spectral range of initial peak lies between $8.8 \mathrm{kHz}$ and 62 $\mathrm{kHz}$ with the average spread distribution of $13.6 \mathrm{kHz}$ to about $30 \mathrm{kHz}$, whereas the spectral range of ramp is from $5.5 \mathrm{kHz} 81 \mathrm{kHz}$ with average spread distribution of $8 \mathrm{kHz}$ to $34 \mathrm{kHz}$. The amplitude of the peak energy radiated by the initial stage is less than that of ramp with an average ratio of 0.5 :

Table 1. Statistics of the wavelet power spectrum of positive return strokes

\begin{tabular}{|c|c|c|c|c|c|c|}
\hline Statistics & $\begin{array}{c}\text { Spectral } \\
\text { range for the } \\
\text { Initial stage } \\
(\mathrm{kHz})\end{array}$ & $\begin{array}{c}\text { Spread } \\
\text { distribution of } \\
\text { initial stage } \\
(\mathrm{kHz})\end{array}$ & $\begin{array}{c}\text { Spectral range } \\
\text { for the ramp } \\
(\mathrm{kHz})\end{array}$ & $\begin{array}{c}\text { Spread } \\
\text { distribution } \\
\text { of ramp } \\
(\mathrm{kHz})\end{array}$ & $\begin{array}{l}\text { Power peak for } \\
\text { the Initial stage } \\
\qquad\left(\mathrm{V}^{2} / \mathrm{m}^{2}\right)\end{array}$ & $\begin{array}{l}\text { Power peak for } \\
\text { the ramp }\left(\mathrm{V}^{2} / \mathrm{m}^{2}\right)\end{array}$ \\
\hline Minimum & 8 & 9 & 4 & 6 & 4000 & 9500 \\
\hline Maximum & 80 & 40 & 100 & 60 & 7000 & 13000 \\
\hline $\begin{array}{l}\text { Average } \\
\text { range }\end{array}$ & $8.8-62$ & $13.6-30$ & $5.5-81$ & $8-34$ & 5600 & 11180 \\
\hline
\end{tabular}

The power radiated by the small pulses superimposed in the ramp is overwhelmed by the low frequency radiation. An example of wavelet power spectrum of a positive return stroke is shown in fig 3.

\section{DISCUSSION AND CONCLUSION}

The electric fields due to the positive lightning flashes striking the sea and propagating entirely over the salt water (except for last few tens of meters) have been 
recorded and analyzed to study the spectral variation of positive ground flashes. Flat plate antenna was system, a broad band antenna system, was employed to sense the vertical electric fields. The electric fields so sensed were acquired in an oscilloscope (digitizer) with a sampling rate of $100 \mathrm{MS} / \mathrm{s}$. The digitized time domain electric field signatures pertinent to the positive ground flashes have been analyzed in frequency domain using two mathematical tools namely Fourier transform and wavelet transform techniques. From the frequency spectrum obtained with the Fourier transform technique it can be observed that the positive return strokes possess maximum energy at or below $10 \mathrm{kHz}$ the amplitude of the energy spectra decreases with the increase in the frequency.

The amplitude of the average energy spectral density of the positive return strokes is found to apparently decrease as $f^{-1}$ from $10 \mathrm{kHz}$ to $0.1 \mathrm{MHz}$, nearly as $f^{-2}$ from $0.1 \mathrm{MHz}$ to about $8 \mathrm{MHz}$ and apparently remains constant afterwards with a few peaks at $12 \mathrm{MHz}, 15 \mathrm{MHz}$, and 19 $\mathrm{MHz}$.

On the other hand, from the frequency spectrum obtained with wavelet transform technique it can be seen that the positive return stroke radiates in the frequency range of 4 $\mathrm{kHz}$ to $100 \mathrm{kHz}$ mostly being contributed by the ramp portion of the return stroke. The maximum energy due to ramp portion is distributed in the frequency range of $6 \mathrm{kHz}$ to $60 \mathrm{kHz}$. Whereas, the initial portion of the return stroke radiates in the frequency range of $8 \mathrm{kHz}$ to $80 \mathrm{kHz}$ and maximum energy is being distributed in the frequency range of $9 \mathrm{kHz}$ to $40 \mathrm{kHz}$.

From the results obtained from the two techniques, it can be seen that, unlike the Fourier transform which shows the variation of power with frequency, the wavelet transform shows the most dominant frequency content of the wave. From the wavelet transform one can easily tell the frequency localization of the maximum power of radiation. Consequently, the variation of power with frequency should show maximum peaks at different frequencies for different lightning events. Such an information cannot be obtained with Fourier transform technique. Wavelet transform is therefore, a powerful tool not only to identify the power content of the wave in timefrequency space but also to localize the power in the frequency space. The information about the frequency content of the electric and magnetic fields due to lightning is very important for the design engineers to design the appropriate protective measures and scientists to understand the physical process that results in the radiation of such fields. The frequency content without losing the time domain information is of extremely importance in order to understand the physics of discharge at various phases lightning discharge that can be achieved by the wavelet transform and is not possible with Fourier transform technique.

Therefore, the wavelet transform technique is very power tool to extract frequency content of a non-stationary signal without losing the time domain information unlike that in the Fourier transform. However, care has to be taken to select the nature of mother wavelet so that it can imitate the signal under investigation.

Frequency spectrum pertinent to the lightning positive return strokes is very scarce in the literature. Furthermore, to the best of our knowledge this is the first study that has employed both Fourier Transform and Wavelet technique to extract the frequency spectrum of the same lightning signal. It is therefore, this study is the first of its kind to detect the frequency of the lightning utilizing the two mathematical tools.

\section{SCOPE OF FUTURE WORK}

The frequency spectrum of the radiative fields due to lightning strikes is of much interest to both physicists and design engineers, to understand the nature of the discharges and to design the appropriate protective system. Availing the techniques that have been used in this study, to various types of lightning discharges such as negative return strokes, cloud discharges, narrow bipolar discharges, initial breakdown pulses and several other discharges, the researcher might be able to understand the discharge phenomena that has not yet been fully understood. Furthermore, as of today, the design engineers are able to design protective system that could provide accurate protective measures owing to the lack of 
complete information on the signatures of various kinds of lightning discharges. As a future work, it is recommended to detect the lightning discharges both with wide band antenna system and narrow band antenna system and then to employ wavelet technique to extract the frequency content of such radiation.

\section{ACKNOWLEDGEMENTS}

Author would like to acknowledge the International Science Programs (ISP), Uppsala University for their financial support for the devices. Author wish to thank Department of Electricity and Lightning Research, Uppsala University, Sweden for providing the excellent research facilities. Author would like to highly acknowledge the Erasmus Mundus 'EXPERT4Asia' program for the financial support and fellowship provided to carry out this research.

\section{REFERENCES}

1. Cooper M. A. and Holle R. L. 2019. Reducing Lightning Injuries Worldwide, Springer Natural Hazards. Springer International Publishing. ISSN 2365-0656, ISBN 978-3-319-77561-6: 3-4. doi.org/10.1007/978-3-319-77563-0.

2. Weidman C. D., Krider E. P. and Uman M. A. 1981. Lightning amplitude spectra of lightning radiation fields in the interval from $100 \mathrm{kHz}$ to $20 \mathrm{MHz}$. Geophys. Res. Lett. 8: 931-934.

3. Willett, J.C., Bailey, J.C., Leteinturier C., and Krider, E.P. 1990. Lightning electromagnetic radiation field spectra in the interval from 0.2 To 20 MHz. J. Geophys. Res. 95: 20 367-87.

4. Le Vine D. M.1987. Review measurements of the RF spectrum of radiation from lightning., Meteorol. Atmos. Phys. 37:195- 204.

5. Nanevicz J. E., Vance E. F. and Hamm J. M. 1987. Observation of Lightning in the frequency and time domains. Lightning Electromagnetics, ed. Robert Gardner. 7: 267-286.

6. Serhan G. I., Uman M. A., Childers D. G., and Lin Y. T. 1980. The RF spectra of first and subsequent lightning return strokes in the 1to 200- km range. Radio Sci. 15: 1089-1094.

7. Weidman C. D. and Krider E. P. 1986. The amplitude spectra of lightning radiation fields in the interval from 1 to $20 \mathrm{MHz}$. Radio Sci., 21: 964-970.

8. Torrence, C., and G.P. Compo. 1998. A practical guide to wavelet analysis. Bulletin of American Meteorological Society. 79: 61-78.

9. Miranda, F. J. 2008. Wavelet analysis of lightning return stroke. Journal of Atmospheric and Solar-Terrestrial Physics. 70(11): 1401-1407.

10. Sheshyekani K., Hazrati, M., Sattari, P., Sadeghi, S.H.H., Moini, R. 2006. Real-Time Detection of Lightning Electromagnetic Field Data: A Wavelet Approach. Proceedings of the 17th International
Zurich Symposium on Electromagnetic Compatibility (EMC Zurich 2006), Singapore.

11. Santamaria, F.; Cortés, C. A. and Román, F. 2009. Noise reduction of measured lightning electric fields signals using the wavelet transform. X International Symposium on Lightning Protection. Curitiba, Brazil, $9^{\text {th }}-13^{\text {th }}$ November 2009.

12. Xueqiang G., Mingli C., Yjun Z., Wansheng D. and Xiushu Q., 2009. Wavelet multi- resolution based multifractal analysis of electric fields by lightning return strokes. Atmospheric Research. 91: 410-415.

13. Nedjah O., Hussein A.M., Krishnan S., Sotudeh R., 2010. Comparative study of adaptive techniques for denoising $\mathrm{CN}$ Tower lightning current derivative signals. Digital Signal Processing. 20: 607-618.

14. Misiti M., Misiti Y., Oppenheim G. and Poggi, J. M.; 2002 Wavelet tool box, User's guide, version 2. The Math Works, Inc.

15. Sharma, S. R., Cooray V., Fernando M., Miranda F. J. 2011. Temporal features of different lightning events revealed from wavelet transform. Journal of Atmospheric and Solar-Terrestrial Physics. 73(4): 507-515.

16. Sharma S. R., Fernando M. and Gomes C. 2005. Signatures of electric field pulses generated by cloud flashes, Journal of Atmospheric and Solar-Terrestrial Physics. 67: 413-422, doi.org/10.1016/j.jastp.2004.09.007.

17. Cooray V. 2003. "The Lightning Flash", Published by The Institute of Electrical Engineers (IEE), London ed. V. Cooray.

18. Gomes C., Cooray V. 1998. "Long impulse currents associated with positive return strokes". J. Atms. Solar-Terr. Phys. 60: 693-699

19. Graps A. 1995. "An introduction to wavelets," IEEE Comput. Sci. Eng. 2.2: 50-61. 